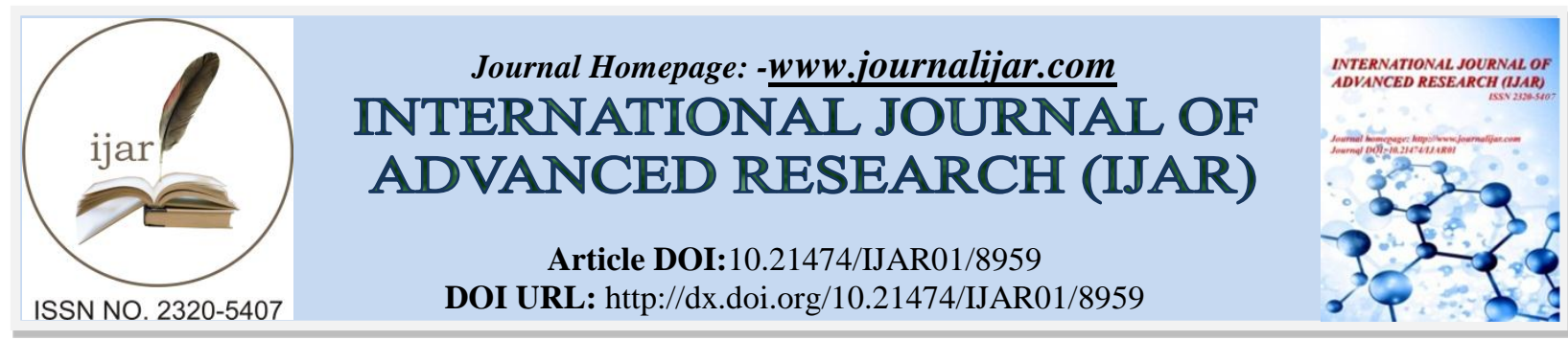

RESEARCH ARTICLE

\title{
DIAGNOSTIC UTILITY OF SHORT AXIS METHOD OF HEART DISSECTION VERSUS INFLOW OUTFLOW METHOD IN SUDDEN CARDIAC DEATH.
}

Priyanka Agasimani $^{1}$, Dayananda S Biligi ${ }^{2}$, A.M.Patil ${ }^{3}$ and Saeed Yendigeri ${ }^{3}$.

1. Assistant Professor, Department of Pathology, Al Ameen Medical College \& Hospital, Athani Road, Vijayapura-586108,Karnataka, India.

2. Professor, Department of Pathology, Bangalore Medical College and Research Institute, Fort, K R Road, Bangalore-560002.

3. Professors, Department of Pathology, Al Ameen Medical College \& Hospital, Athani Road, Vijayapura586108,Karnataka, India.

\section{Manuscript Info}

\section{Manuscript History}

Received: 22 February 2019

Final Accepted: 24 March 2019

Published: April 2019

\section{Key words:-}

Sudden cardiac death, Coronary artery disease, Triphenyl tetrazolium chloride Masson trichrome and Heart dissection.

\section{Abstract}

Background and Objectives:Cardiac pathology is responsible for $80 \%$ of sudden deaths. Inflow outflow method is the most commonly used method of heart dissection which often fails to find out cause of death. Triphenyl tetrazolium chloride staining is used for macroscopic diagnosis of early infarcts. Objectives of the study was to assess the diagnostic utility of short axis method over inflow outflow method of heart dissection, to assess the necessity of Triphenyl tetrazolium chloride staining to pick up acute MI of 2-6hrs.

Methods:60 cases of sudden cardiac deaths were studied in Department of Pathology, Bangalore Medical College \& Research Institute, Bangalore. 30 cases were dissected by inflow outflow method and 30 cases were dissected by short axis method. Triphenytetrazolium chloride staining was done on fresh hearts to detect early infarcts. Morphometric analysis was done to assess myocyte diameter in case of HCM.

Results:Study included 56 males and 4 females. The commonest cause of sudden cardiac death in our study was ischaemic heart disease (50\%), non-ischaemic sudden cardiac deaths occurred in hypertrophic obstructive Cardiomyopathy (8.3\%), arrythmogenic right ventricular dysplasia (1.6\%), mitral valve prolapse (1.6), mitral stenosis (5\%), coronary artery dissection (1.6\%) and others (8.3\%). Triphenyl tetrazolium chloride showed infarcts grossly which was confirmed by histology.

Interpretation and Conclusions:Short axis method of heart dissection is better method for finding infarcts, cardiomyopathies, biventricular hypertrophies and myocarditis. Triphenyl tetrazolium chloride staining is sensitive and specific method for finding out early infarcts. Masson trichrome is excellent in finding out fibrosis in case of HCM and chronic IHD. 


\section{Introduction:-}

The vast majority of naturally occurring sudden deaths are caused by cardiac disorders. As per WHO census statistics, mortality due to cardiac cause has overtaken mortality due to all cancer put together. 4280 out of every 1 lakh people die every year from sudden cardiac death in India alone. Sudden cardiac death accounts for 3,00,000 to 4,00,000 death annually in US. ${ }^{1}$

$80 \%$ of sudden cardiac deaths in individuals $>35 \mathrm{yrs}$ is due to ischemic heart disease, followed by hypertrophic cardiomyopathy (5\%), valvular heart disease (5\%), mitral valve prolapse (5\%), unexplained (5\%) whereas in individuals <35years of age hypertrophic cardiomyopathy accounts for $48 \%$ of sudden cardiac death. ${ }^{2}$

Cardiomyopathies represent second largest group of patients who experience sudden cardiac death. ${ }^{3}$ The incidence of hypertrophic cardiomyopathy in United states is low and accounts 0.02 to 0.2 percent of the population and is found to be in $0.5 \%$ of un-selected patients referred for an echocardiographic examination. ${ }^{4}$ In Japan the prevalence per 100,000 population is 17.3 which is same as in the Western population. ${ }^{5}$ The condition is being increasingly recognised in India and yet, there is little data available regarding the incidence and the rates of mortality. ${ }^{6}$

The conventional method of heart dissection is inflow outflow method. The disadvantage of this method is, it will not expose completely the left ventricular wall, right ventricular wall and the interventricular septum. Thus fails to demonstrate satisfactory clinicopathological correlation in cases of sudden cardiac death. The reason being isolated interventricular septal thickening in cases of Cardiomyopathies and myocardial infarction due to obstruction of right coronaries. Arrythmogenic right ventricular dysplasias are also emerging as important cause of sudden cardiac death which also needs exposure of right ventricle.

To overcome above deficiencies we used short axis method. By doing short axis method we exposed the entire circumference and the thickness of left ventricle, right ventricle and interventricular septum. This is the best technique for inspecting the myocardium for infarcts. $7,8,9 \& 10$

Thus a study to evaluate diagnostic utility of short axis method of heart dissection over inflow outflow method.

Triphenyl tetrazolium chloride helps us to pick up ischemic change as early as 2-6hrs. ${ }^{11}$ Masson trichrome stain helps us to demonstrate the interstitial fibrosis in cases of cardiomyopathies and chronic ischemic heart disease. ${ }^{12}$ Morphometry of myocardial fibre will aid in assessing the hypertrophy of individual fibres. This is an attempt to utilize the above parameters to increase satisfactory clinicopathological correlation in sudden death.

\section{Materials and methods:-}

The present study emphasises the diagnostic utility of short axis method of heart dissection over inflow outflow method.

Ours was a prospective study conducted on specimen of heart of sudden death cases suspected of cardiac origin submitted to the department of Pathology from forensic medicine Unit of Victoria Hospital and Bowring and Lady Curzon hospital, Bangalore Medical College \& Research Institute, Bangalore,. The study was commenced after obtaining required approvals and clearances from the institution ethical committee. 60 cases of sudden death suspected of cardiac origin was studied during the period of October 2013 to May 2015.

Cases were included only after having met the inclusion and exclusion criteria mentioned for this study. On arrival to the department, the specimens were adequately fixed in $10 \%$ formalin following which the specimens were grossed.

The first 30 hearts were dissected by inflow-outflow method in our department. For each side of the heart, the atrium is opened first, and then the ventricle is opened along its inflow and outflow tracts, following the direction of blood flow. And next 30 hearts were dissected by short axis method with transverse slicing of hearts $1-1.5 \mathrm{~cm}$ from apex to base of heart. The coronary arteries were cut in cross sections at 3-5 mm intervals and examined grossly for presence of any atherosclerosis, thrombus and narrowing. 
Sections were taken from coronaries, right coronary artery, left coronary artery, left circumflex and left anterior descending artery. Right and left ventricle wall thickness was measured and sections taken. Sections were also taken from major vessels, aorta and pulmonary artery. Section from interventricular septum was taken in case of short axis method and then subjected to routine processing for paraffin embedding, stained with Haematoxylin and Eosin $(\mathrm{H} \&$ E) method according to standard procedures.

Masson trichrome staining was done in case of HCM and chronic IHD. This helps us to demonstrate the interstitial fibrosis.

Triphenyl tetrazolium chloride staining was done on 25 fresh hearts of sudden death cases suspected of cardiac origin after meeting the inclusion and exclusion criteria.

\section{Inclusion criteria:}

All cases of sudden death suspected of cardiac origin occurring in the study period.

\section{Exclusion criteria:}

Decomposed bodies where autopsy was not possible within $24 \mathrm{hrs}$ or without proper cold storage.

\section{Results:-}

A total number of 60 cases of sudden death suspected of cardiac origin were studied prospectively from November 2013 to May 2015 in the Department of Pathology and Department of forensic medicine Bangalore Medical College and Research Institute, Bangalore

The observations of this study are as follows-

Age distribution:

Among 60 sudden cardiac death cases youngest was 17years and oldest being 58year.

Figure 1:-Graph representing Age-wise Distribution of cases $(n=60)$

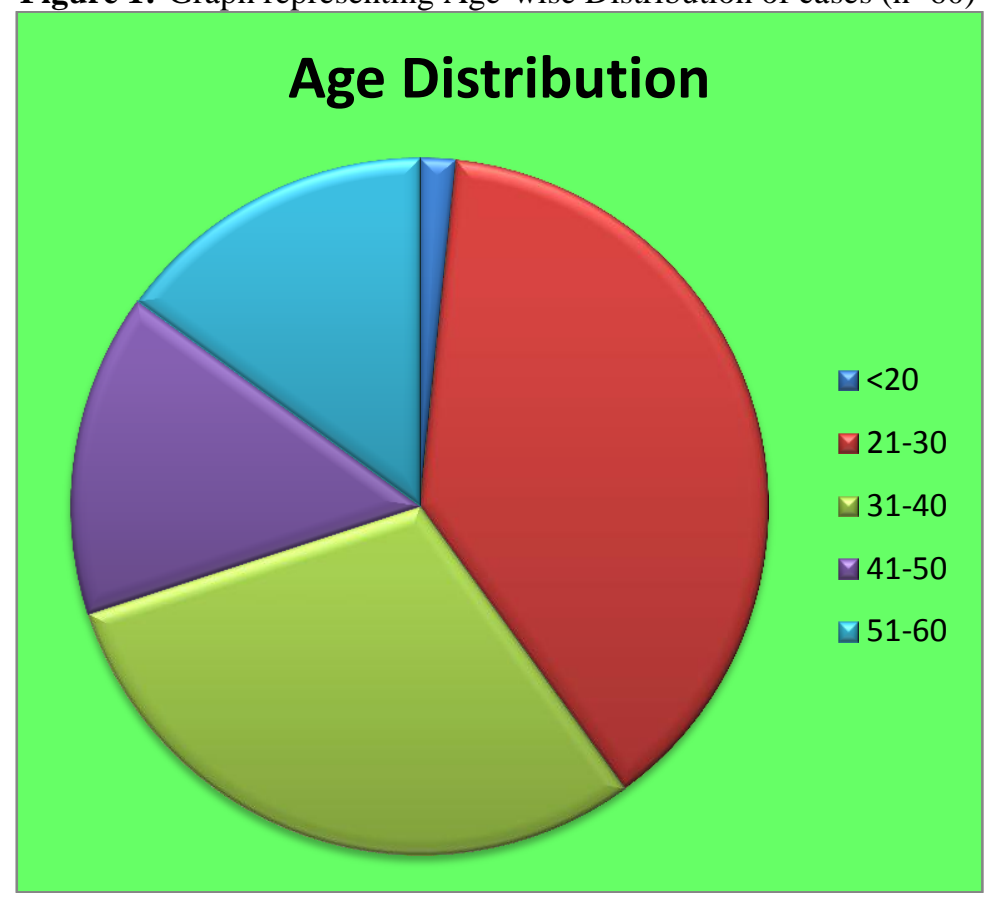

Table 1:-Showing distribution of various cardiac diseases

\begin{tabular}{|l|l|l|l|}
\hline Sl. No & Cardiac disease & No. of cases & Percentage (\%) \\
\hline 1. & Ischemic heart disease/coronary artery disease & 30 & 65.6 \\
\hline 2. & Hypertrophic cardiomyopathy & 05 & 10.8 \\
\hline
\end{tabular}




\begin{tabular}{|l|l|l|l|}
\hline 3. & Mitral stenosis & 03 & 6.5 \\
\hline 4. & Myxomatous degeneration of mitral valve & 01 & 2.1 \\
\hline 5. & Arrythmogenic right ventricular dysplasia & 01 & 2.1 \\
\hline 6. & Coronary artery dissection & 01 & 2.1 \\
\hline 7. & Others & 05 & 10.8 \\
\hline & Total & 46 & 100 \\
\hline
\end{tabular}

$65 \%$ of sudden cardiac death was due to ischemic heart disease and hypertrophic cardiomyopathy accounted for $10 \%$ of cases.

Table 2:-Showing comparison of short axis method of heart dissection with inflow outflow method:

\begin{tabular}{|l|l|l|l|l|}
\hline Sl. No & Cardiac disease & No. of cases & Total \\
\hline & & Inflow outflow & Short axis & \\
\hline 1. & Ischemic heart disease/coronary artery disease & 17 & 13 & 30 \\
& & & & \\
\hline 2. & Hypertrophic cardiomyopathy & 02 & 05 & 05 \\
\hline 3. & Mitral stenosis & 01 & 02 & 03 \\
\hline 4. & Myxomatous degeneration of mitral valve & 01 & 00 & 01 \\
\hline 5. & Arrythmogenic right ventricular dysplasia & 00 & 01 & 01 \\
\hline 6. & Coronary artery dissection & 00 & 01 & 01 \\
\hline 7. & Others & 00 & 05 & 05 \\
\hline & Total & 21 & 25 & 46 \\
\hline
\end{tabular}

Out of 30 IHD grossly infarct was seen in 13 cases. Short axis method of heart dissection was better in identifying infarct grossly compared to inflow outflow method.

\section{Distribution of cardiomyopathies}

There were no much differences in the two methods in finding out cardiomyopathies. Out of $5 \mathrm{HCM}$, 3cases were identified by short axis method. May be occurring of the disease was just by chance.

The 5 cases of HCM accounted for $8.3 \%$ of the cases of sudden cardiac death. HCM was seen in younger individuals (20-30years). out of which 4 were male and 1 female.

Table 3:-Showing cardiac weight, left ventricular thickness and average myocyte .

\begin{tabular}{|l|l|l|l|}
\hline Case no & Cardiac weight in $\mathrm{kg}$ & Left ventricle wall thickness in cms & Average myocyte in microns \\
\hline 1. & 540 & 3.0 & 93.5 \\
\hline 2. & 500 & 2.5 & 72.5 \\
\hline 3. & 450 & 2.2 & 105.1 \\
\hline 4. & 350 & 2.0 & 98.2 \\
\hline 5. & 325 & 1.6 & 94.3 \\
\hline
\end{tabular}

Above table shows that increase in cardiac weight is associated with increase in left ventricular wall thickness. Increase in cardiac weight is also associated with increase in myocyte length. Interstitial fibrosis is associated with reduced myocyte length.

Triphenyl tetrazolium chloride staining of heart:

Triphenyl tetrazolium chloride staining was done on 25 fresh hearts at autopsy. Out of which 17 were positive and 8 negative. Among 16 positive cases 13 showed features of infarction histologically. 3cases did not show features of infarction histologically. 
Figure 2:-Graph representing result of TTC and H\&E stain of myocardium slice

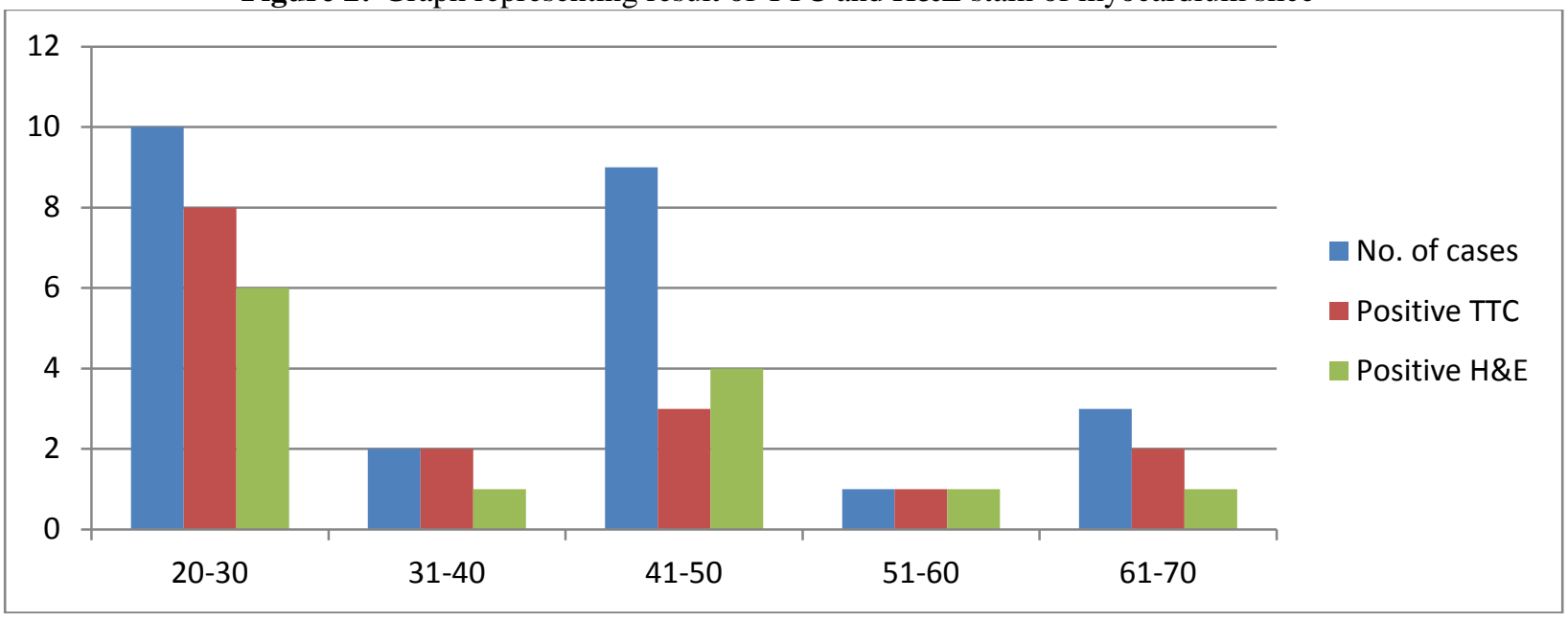

Out of 17 TTC positive cases, grossly infarct was seen in 9cases which was confirmed by TTC staining. 8 cases showed infarct only after staining with TTC, this shows that TTC staining is better in picking up infarcts.

Table 4:-Showing comparision of gross, microscopy and ttc staining

\begin{tabular}{|l|l|l|l|l|l|}
\hline Sl.no & Age in years & No. of cases & Gross & Positive TTC & Microscopy \\
\hline 1. & $20-30$ & 10 & 03 & 08 & 07 \\
\hline 2. & $31-40$ & 02 & 00 & 02 & 01 \\
\hline 3. & $41-50$ & 09 & 04 & 03 & 03 \\
\hline 4. & $51-60$ & 01 & 01 & 01 & 01 \\
\hline 5. & $61-70$ & 03 & 01 & 02 & 01 \\
\hline & Total & 25 & 09 & 16 & 13 \\
\hline
\end{tabular}

\section{Discussion:-}

Sudden cardiac death is commonly defined as an unexpected natural death due to cardiac cause within a short time period (usually within one hour) with or without onset of symptoms and without any prior conditions that would appear fatal. ${ }^{13}$

Cardiac pathology is responsible for $80 \%$ of sudden deaths and therefore presently, largest numbers of histopathological studies are being carried out on whole heart ${ }^{14}$. Heart disease at autopsy necessitates the careful preservation and examination of the heart and the vessels. Techniques preserving these and using a reproducible and systematic approach are preferred. ${ }^{15}$.

The commonly performed autopsy of the heart often fails to demonstrate a satisfactory clinicopathological correlation in cases of death due to ischemic heart disease. ${ }^{6}$

Since most of causes of death are cardiac it is essential that all pathologists are familiar with the approach to dissection of the heart. ${ }^{16}$

According to Horn et al, The Rokitansky method of organ block dissection, in combination with a system of heart examination termed sequential segmental analysis provides a systematic approach in careful preservation and examination of the heart. He also says sequential method of examining the heart enables documentation of even the most complex cardiovascular anomalies. ${ }^{15}$

Our study was to compare short axis method of heart dissection with inflow outflow method. Very limited studies are available comparing heart dissection methods.

In our present study we found that short axis method of heart dissection was better in identifying infarcts grossly. But overall we found more cases of ischemic heart disease by inflow outflow method. Short axis method was better 
in finding out cardiomyopathies (HCM and ARVD), myocarditis and ventricular hypertrophies as it exposes maximum surface area of ventricular myocardium.

According to Vittorio Fineschi et al, histology may offer structural details of the cardiac wall and coronary intraluminal changes, particularly when serial section studies are performed. ${ }^{17}$

As stated by Alexandre de et al, the macroscopic diagnosis of Acute MI was considered mainly during the examination of transverse ventricular sections. ${ }^{18}$ Transverse slicing of myocardium permits best identification of infarction as well as easy location and documentation of grossly discernible myocardial infarcts. ${ }^{11}$

The present study was carried out on 60 autopsy cases that included 56 males and 04 females. The age group in this study ranged from 17 to 60 years. In present study, the percentage of cardiac cases below 40 years was $72 \%$ which is closer to study conducted by M Ahmad et al $(71 \%){ }^{1}$

Overall, the commonest cause of sudden cardiac death in our study was ischaemic heart disease, non-ischaemic sudden cardiac deaths occurred in hypertrophic obstructive cardiomyopathy, arrythmogenic right ventricular dysplasia, mitral valve prolapse, mitral stenosis, coronary artery dissection and myocarditis. In our study, coronary artery disease accounted for $64 \%$.

In a clinicopathological study of sudden death by Kasthuri et al,76.9\% individuals died of coronary artery diseases. ${ }^{20}$

When sudden death $(\mathrm{SD})$ occurs in adults and elderly persons, coronary atherosclerosis is the usual cause. ${ }^{21}$ On the contrary, a large spectrum of cardiovascular diseases, both congenital and acquired, may account for SD in the young 21

In our study coronary atherosclerosis was leading cause of death in elderly persons. $72 \%$ of our cases were $<40$ years. In them also the most common cause of death was coronary artery disease which accounted to $45 \%$. In Drory et al. study coronary artery disease accounted to $58 \%$ in young individuals (<40years) which is similar to our study. ${ }^{22}$

In a study done by Basso et $\mathrm{al}^{21}$, the most common cause of sudden death in young included premature coronary atherosclerosis $(21 \%)$, ARVC $(14 \%)$, mitral valve prolapse $(12 \%)$, non-atherosclerotic coronary artery, disease $(11 \%)$, myocarditis (10\%), conduction system disease $(9 \%)$ and HCM $(7 \%)$.

In the present study five young individuals (21-30years) revealed gross and microscopic features of hypertrophic cardiomypathy which is similar study done by M Ahmad et al. which is also similar to two other studies done by Mckenna $^{23}$ and Nocod $^{24}$

Table 5:-Showing age distribution of HCM in various studies

\begin{tabular}{|l|l|l|l|l|l|}
\hline Sl. no & Study & Year & Age distribution & No. of cases & HCM $(\%)$ \\
\hline 1. & Drory et al & 1985 & $9-39$ & 118 & 13 \\
\hline 2. & Kramer et al & 1986 & $17-30$ & 24 & 25 \\
\hline 3. & Basso et al & 1999 & $1-35$ & 273 & 7 \\
\hline 4. & Ahmad et,al, & 1995 & $21-30$ & 245 & 6.1 \\
\hline 5. & Present study & 2015 & $21-30$ & 60 & 8.3 \\
\hline
\end{tabular}

In present study hypertrophic cardiomyopathy accounted for $8.3 \%$ of sudden cardiac deaths which was in close relation to study done by mumtaz et al $(8 \%)^{19}$ and kasthuri et al $(7.6 \%)^{20}$

In a Spanish study ${ }^{25}$, hypertrophic cardiomyopathy was seen in $6.5 \% .^{25}$ In another study by ahmad et al, , hypertrophic cardiomyopathy accounted for $6.1 \%$.

Table 6:-Showing frequency of HCM in various studies.

\begin{tabular}{|l|l|l|l|}
\hline Sl. No & Study & No of cases & frequency \\
\hline
\end{tabular}




\begin{tabular}{|l|l|l|l|}
\hline 1. & present study & 60 & 8.3 \\
\hline 2. & mumtaz et,al, 1995 & 66 & 8.1 \\
\hline 3. & Ahmad et,al,1995 & 245 & 6.1 \\
\hline 4. & kasthuri et al,2002 & 130 & 7.6 \\
\hline 5. & Basso et al,1999 & 273 & 7 \\
\hline
\end{tabular}

In the present study, HCM occurred in young individuals <30years of age, which correlated to study done by luqman et al. ${ }^{19}$ According to study done by Mckenna and Nicod et al, sudden death due to HCM also occured in young individuals. ${ }^{23,24}$

It is said to occur more often in young patients, especially those with a family history. ${ }^{26}$ More recently, hypertrophic cardiomyopathy is inherited as an autosomal dominant trait; roughly half of patients have another family member with HCM. Younger age at diagnosis, marked wall thickness and a family history of HCM increase the frequency that a patient will be gene positive. ${ }^{34}$

As far as sex distribution is concerned male to female ratio was 2.3:1 in a Japanese Study ${ }^{27}$, in our study 4 were male and 1 female. In M. Ahmad et al study all were male.

Undetermined cases of sudden cardiac death accounted for $4 \%$ in M. Ahmad et al study which is in contrary to two previous studies where it was $11.8 \%$ to $16.3 \%{ }^{11}$. In present study undetermined cases were $23 \%$. Such cases may be because of myocardial ischaemia caused by coronary spasm secondary to overdriven adrenergic activity.

The absence of gross as well as microscopic changes before appearance of neutrophil at the scene of infarct, which is estimated to take minimum of 6-8 hours in term of post-infarction survival time, has been a major hurdle in establishing the cause of death in cases of early death due to myocardial infarction.

The detection of enzyme specific to the myocardial cell, in increased quantities in the peripheral blood indicates irreversible myocardial cell death. Even at this stage the morphology of these dead cells remains normal to routine light microscopy. However, the decreased activity of the enzyme in the cell can be demonstrated by histochemical method. Absence of dehydrogenase enzyme activity leads to non deposition of formazan pigment over the infarct area.

Table 7:-Studies on triphenyltetrazolium chloride:

\begin{tabular}{|l|l|l|l|l|}
\hline Sl. no & Study with year & Total & TTZ & H\&E \\
\hline 1. & Present study, 2015 & 25 & 16 & 13 \\
\hline 2. & Banshidhar et al & 100 & 68 & 25 \\
\hline 3. & Doran et al & 25 & 24 & 16 \\
\hline 4. & Adegobyega et al & 638 & 174 & 140 \\
\hline 5. & Jishenbein et al & 54 & 54 & 54 \\
\hline 6. & Alpesh B Bambhaniya et al & 130 & 26 & 17 \\
\hline
\end{tabular}

Present study showed infarcts in $68 \%$ cases which closely correlates with $\mathrm{Agdal}^{28}$ study where he found fresh infarct in $75 \%$ of cases. Banshidhar et al ${ }^{11}$ study revealed infarcts in $82.37 \%$ autopsy cases using TTC stain. There were no false positive cases noted in our study. Similar finding was noted in study by johan et al. Therefore according to our study Triphenyl tetrazolium chloride staining is highly specific.

In present study there were 17 TTC positive test, amongst them 8 specimen of heart did not show infarction on macroscopic examination. After application of TTC, infracted areas were well visualized. In 9 hearts in which macroscopically infracted area were seen, after application of TTC these areas were better delineated. Finding of the present study is in accordance with Neural et al ${ }^{29}$, Nachals and Shnikta ${ }^{30}$, Ramkissoon ${ }^{31}$, Brody et al ${ }^{32}$ and Mc Vie ${ }^{33}$ This study shows that the maximum number of early myocardial infarction can be detected by TTC method. The technique of this procedure is quite simple and easy to perform. The reagents required are not out of the reach (economically) for medium sized laboratories. As regards interpretation of results, the TTC macro-method did not pose any problems. 
One of the major objective of medico legal autopsy is to find the cause of death. As TTC staining method is simple and easy to perform, it can be done at autopsy. The unnecessary wastage of effort and resources on investigations can be saved by just doing this simple test at autopsy. An attempt should be made to establish TTC macro method at all the hospitals including primary centers where the postmortems are conducted.

Fig 3:-Figure showing dissection of heart

a)short axis method

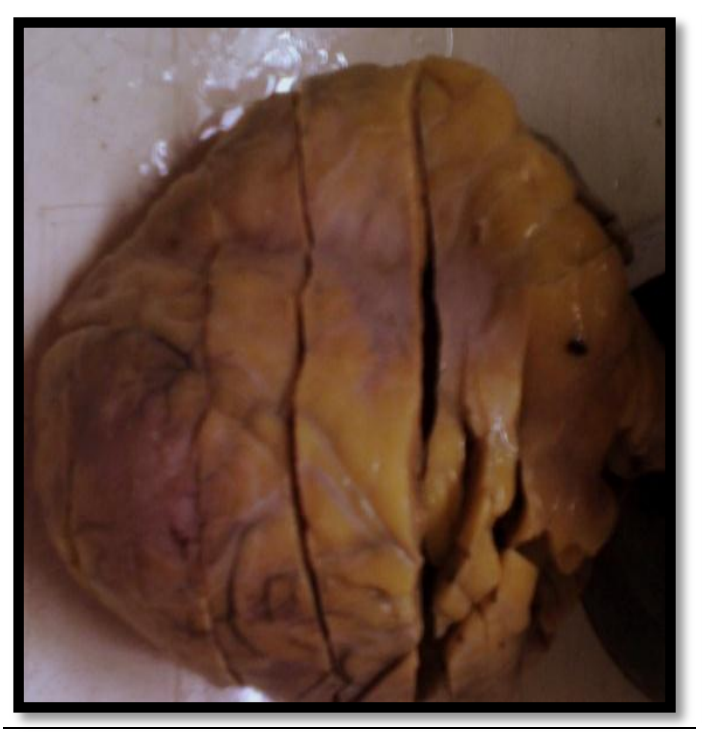

Figure 4a:- infarct at apex

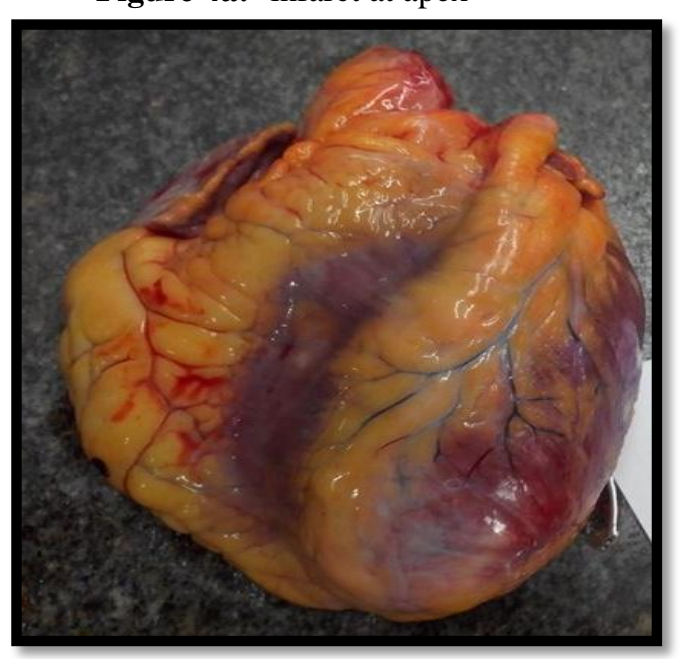

b)inflow outflow method.

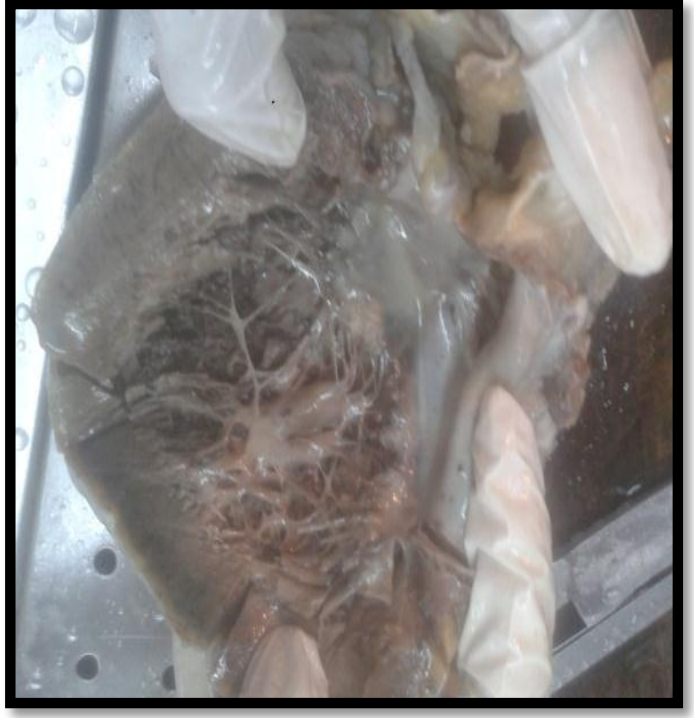

Figure 4b:-coronary artery dissection

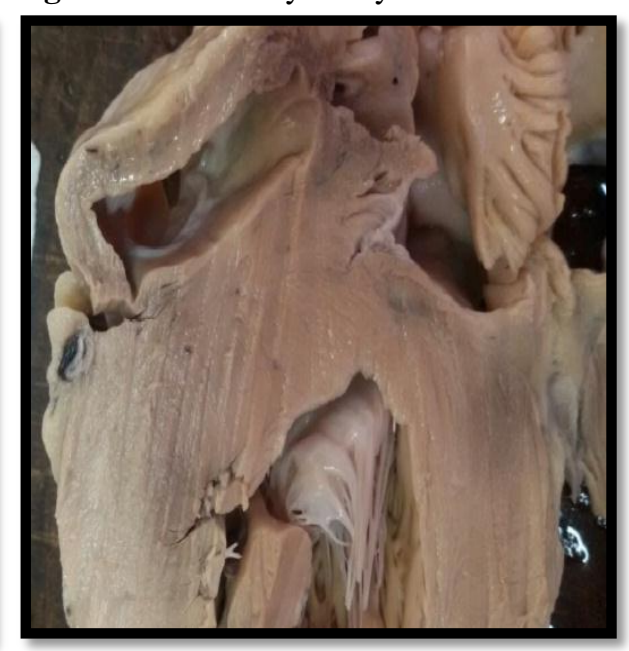


Figure 4c:-Arrythmogenic Right Atrial Dysplasia
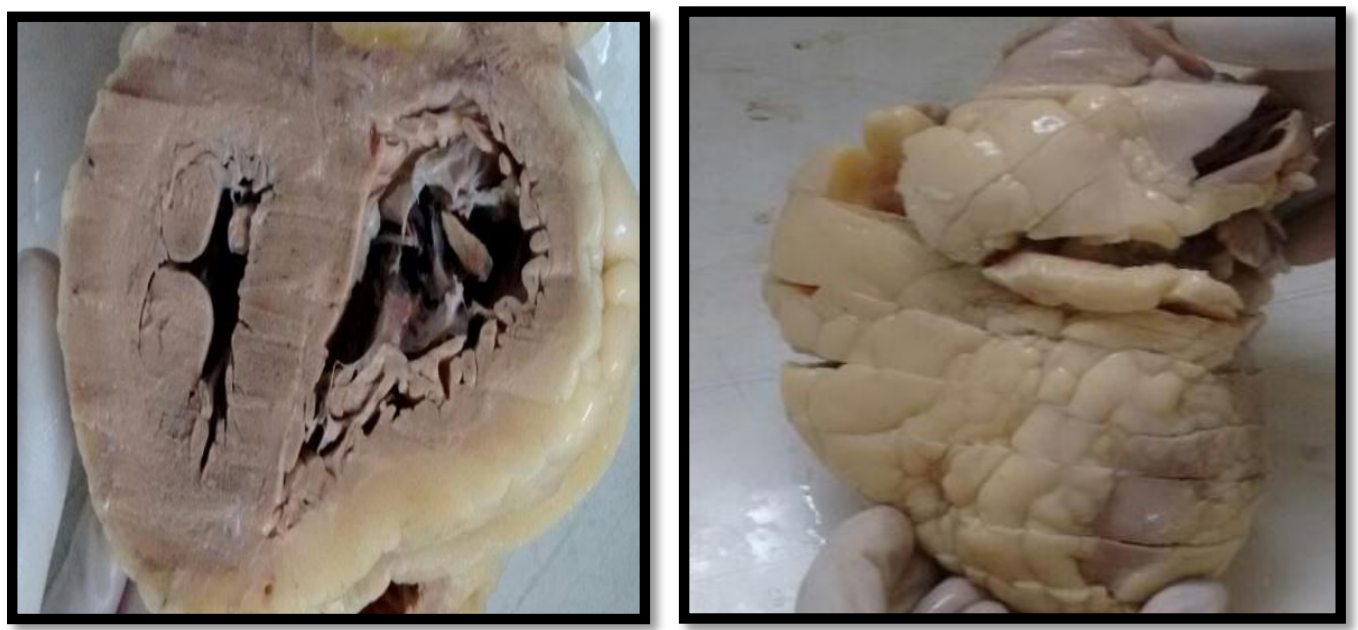

Figure 5a:-TTZ staining control

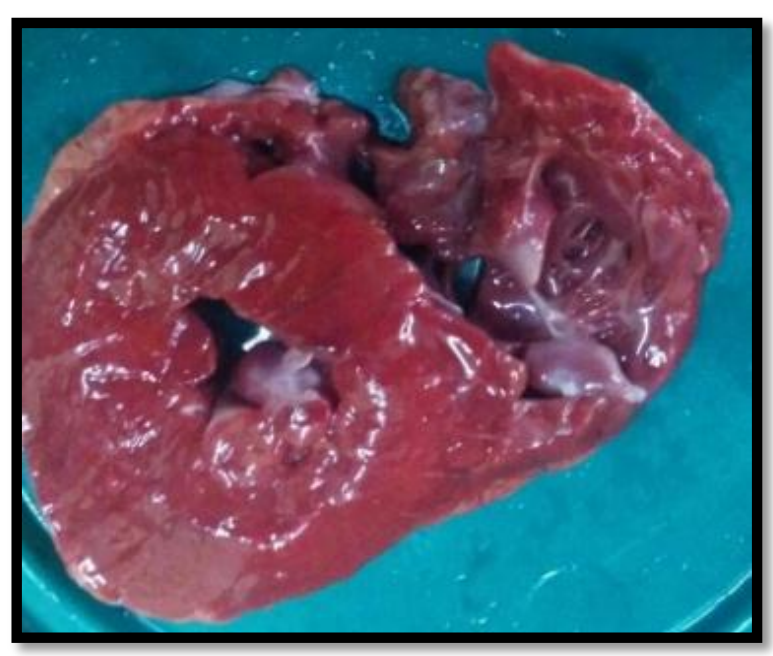

Figure 5b:-TTZ staining showing infarct

Figure 5:-Microscopy of a)Coronary Artery Dissection b)Hypertrophic Cardiomyopathy

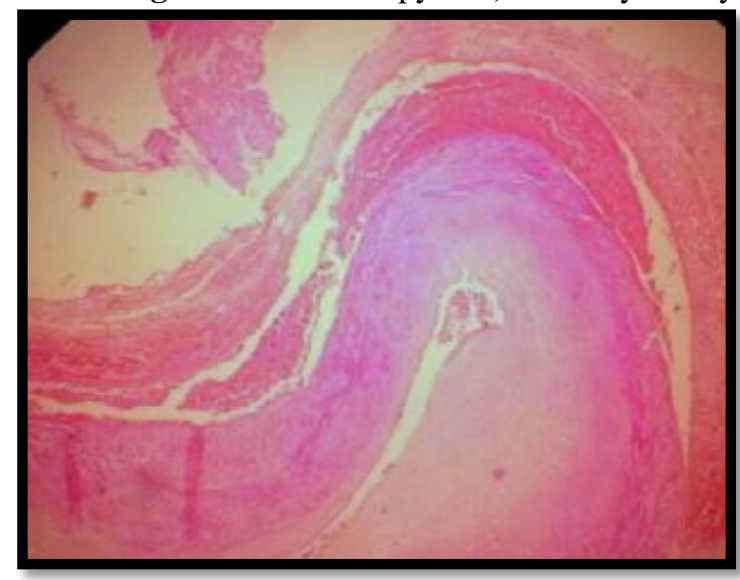

Figure 5c:-Myocardial infarction
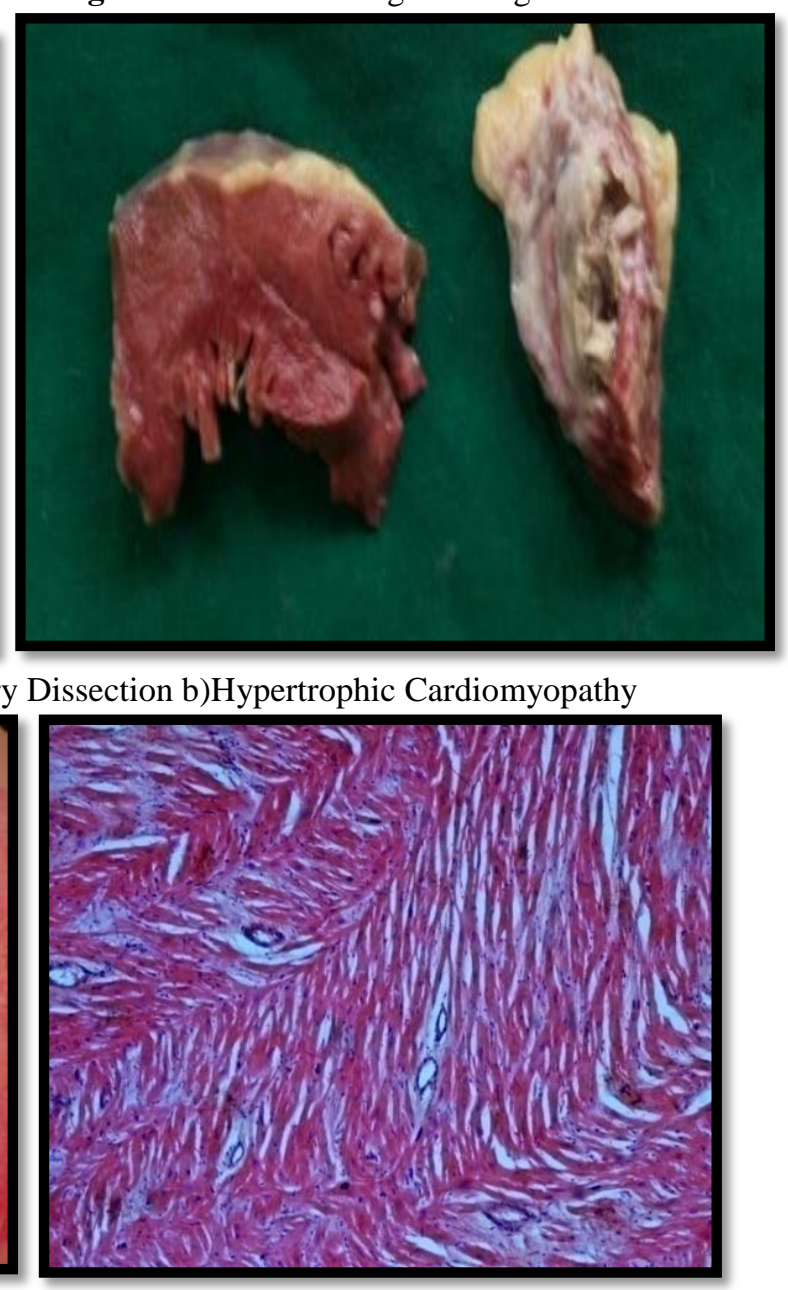

Figure 5d:-ARVD 

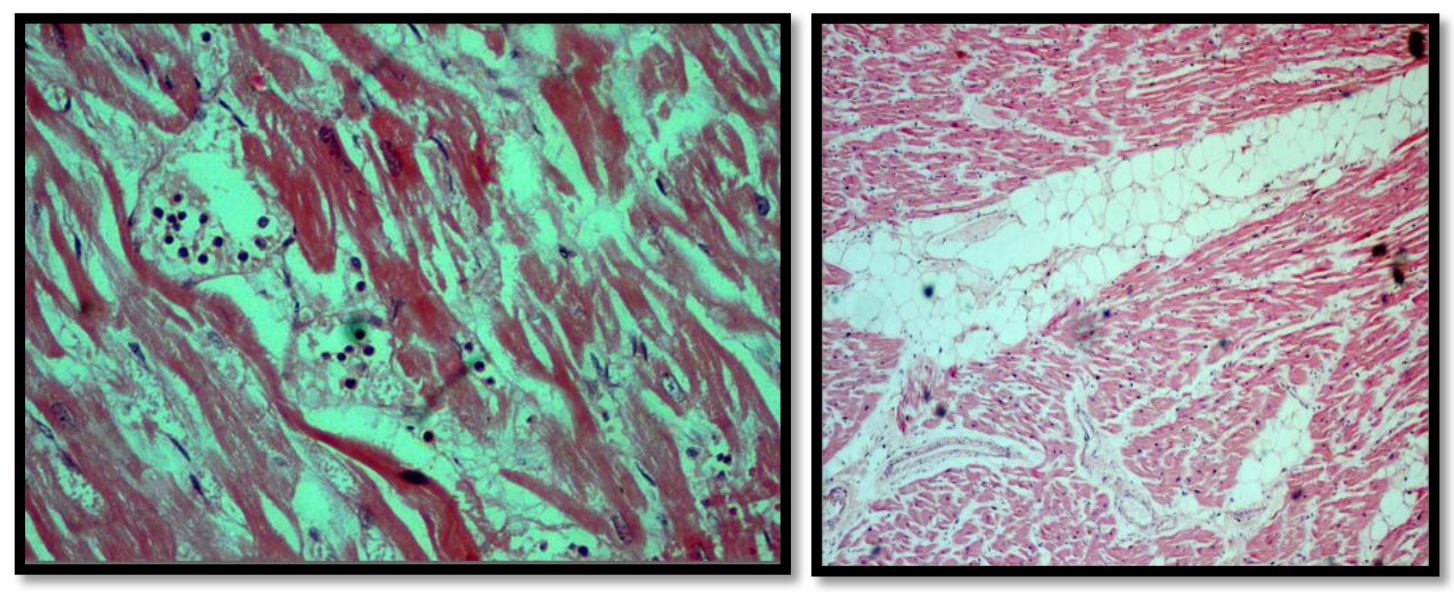

Figure 5e:-Myocyte hypertrophy in HCM

Figure 5f:-Grade II atheroscelosis.
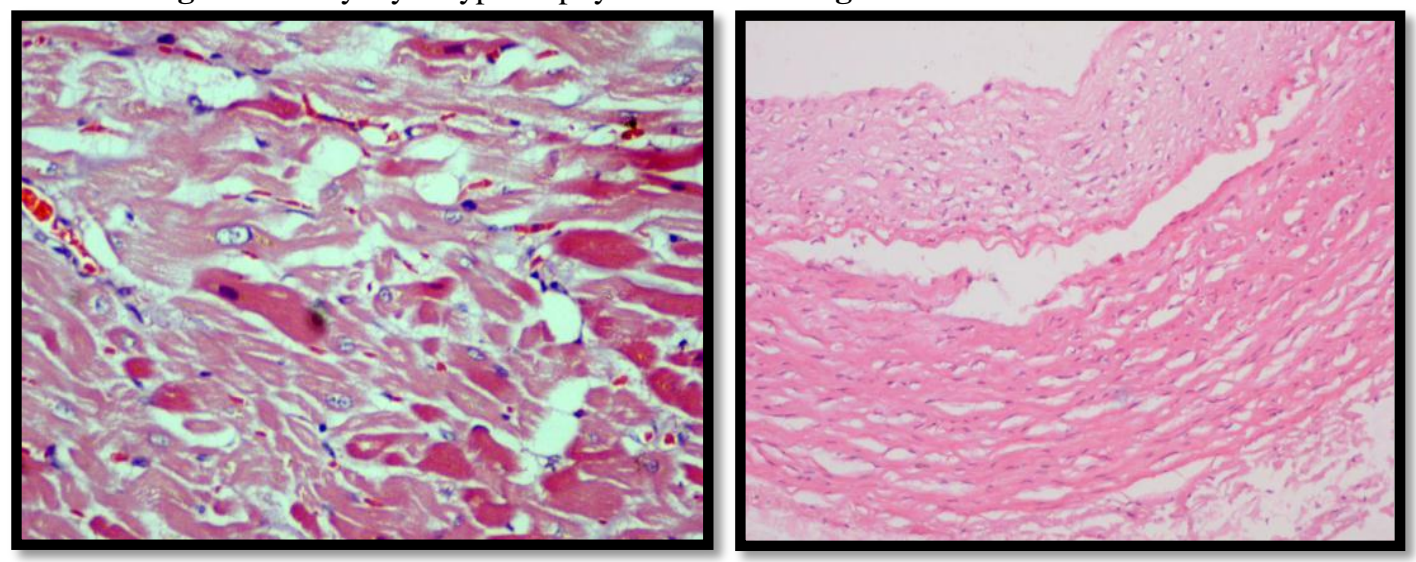

\section{References:-}

1. Engelstein ED, Ziper DP, SCD, In;Alexander RW, Schlant RC, Fuster V, The heart, arteries and veins, New York:McGraw hill, 1998,1081-1112

2. Maron BJ, Epstein SE, Roberts WC; Causes of sudden death in competitive athletes, JACC, 1989;204-214.

3. Spirito P, Seidman CE, Mc Kenna WJ, Management of HCM, N Engl J of medicine, 1997.

4. Maron BJ, Peterson EE, Maron MS et al. Prevalence of hypertrophic cardiomyopathy in an outpatient population referred for echocardiogrpahic study. Am J Cardiol,1994;73:577-80.

5. Miura K, Nakagawa H, Marikawa Y et al. Epidemiology of idiopathic cardiomyopathy in Japan: results from a nationwide survey. Heart 2002;87:126-30.

6. RS Phadke, P Vaideeswar, B Mittal, J Deshpande et al. Hypertrophic cardiomyopathy: an autopsy analysis of 14 cases, J Postgrad Med 47(3),2001:165-170.

7. Finkbeiner, Ursell, Davis, Autopsy Pathology: A Manual and Atlas, $2^{\text {nd }}$ edn, 42.

8. Michael, Deborah, Post mortem technique handbook, $2^{\text {nd }}$ edn, 141-155.

9. Ludwig J, Lie JT. Cardiovascular system. In: Ludwig J, ed. Current Methods of Autopsy Practice, $3^{\text {rd }}$ ed. W.B. Saunders, Philadelphia,PA, 2002;21-44.

10. William H. Westra, MD, et al. Surgical pathology dissection: an illustrated guide, $2^{\text {nd }}$ edn;93-96.

11. Banshidhar Gupta, Hetal Gohel, Nandini Desai, Shital Dodhia et al, post mortem study of heart in cases of sudden cardiac death using triphenyltetrazolium chloride and haemtoxylin and eosin stain, Indian Journals, 21(15b),2013:25-31.

12. Marwah Nisha, Sethi bhawna, Gupta Sumiti, Duhan Amrita, Singh Sunita, Sen Rajeev et al, Histomorphological Spectrum of Various Cardiac Changes in Sudden Death: An Autopsy Study, Iran J Pathol (2011),6 (4), 179-186.

13. Goldstein S (1982) The necessity of a uniform definition of sudden coronary death: witnessed death within $1 \mathrm{~h}$ of the onset of acute symptoms. Am Heart J 103:156-159. 
14. M Paz, Suarez Mier, Beatriz Aguilera, methodological approach to cardiac autopsy, Clinico-Pathological Atlas of Cardiovascular Diseases,2014;1-30.

15. Horn KD, Devine WA, An approach to dissecting the congenitally malformed heart in the forensic autopsy: the value of sequential segmental analysis, Am J Forensic Med Pathol 2001;22(4):405-11.

16. Mary N Sheppard, Approach to the cardiac autopsy, J Clin Pathol 2012;65:484-495.

17. Vittorio Fineschi, Cristoforo Pomara, A Forensic Pathological Approach to Sudden Cardiac Death (1) of the series Forensic Pathology Reviews 139-168.

18. Alexandre de Matos Soeiro, I Aline D, Postmortem diagnosis of acute myocardial infarction in patients with acute respiratory failure - demographics, etiologic and pulmonary histologic analysis, Clinics, 2012;67(3):213217.

19. Luqman M, Sattar A, Abbasi S, Satti TM. Pattern of sudden deaths in armed forces personnel - posmortem study. Pak Armed Forces Med J 1995;45:66-71.

20. Kasthuri AS, Handa A, Niyogi M, Choudhury JC. Sudden death: a clinicopathological study. J Assoc Physicians India 2002;50:551-3.

21. Cristina Basso, Fiorella Calabrese, Domenico Corrado, Gaetano Thiene Postmortem diagnosis in sudden cardiac death victims: macroscopic, microscopic and molecular findings, Cardiovascular Research, 50,2001:290-300.

22. Drory Y, Turetz Y, Hiss Y et al. Sudden unexpected death in persons less than 40 years of age. Am J Cardiol 1991;68:1388-1392.

23. McKenna WJ, Deanfield J, Faruqui A, England D, Oakley C, Goodwin J. Prognosis in hypertrophic cardiomyopathy: role of age, clinical, elctrocardiographic and haemodynamic features. Am J Cardiol 1981; 47: 532-8.

24. Nicod P, Polikar R, Peterson KL. Hypertrophic cardiomyopathy and sudden death. N Eng J Med 1988; 318 : $1255-7$.

25. Suarez-Mier MF, Aguilera B. Causes of sudden death during sports activities in Spain. Rev Esp Cardiol 2002;55:347-58.

26. Hansen BF, Heart autopsy in ischemic heart disease. An autopsy protocol, Acta pathology microbiology scandinavica section A. 1978; 86(3):241-4.

27. Matsumori A, Furukawa Y, Hasegawa K, et al. Epidemiologic and clinical characteristics of cariomyopathies in Japan: results from Nationwide surveys. Circ J 2002;66:323-26.

28. Niels Agdal, Mogens Thyge Anderson The value of succinate dehydrogenase stain in the postmortem diagnosis of early acute myocardial infarction: A forensic study.

29. Neoral L, Kolin A, Kodousek R, Kvasnicka J: A study about early stages of experimental ischemia and myocardial infarction in dogs. Acta Univ. Palak Olomucensis 1956; 11:127-133.

30. Nachlas MM, Shinka TK: Macroscopic identification of early myocardial infarction by alteration in dehydrogenase activity. Am Pathol. 1963; 42:379-405.

31. Ramkissoon RA: Macroscopic identification of early myocardial infarction by alteration in dehydrogenase activity. J Clin Pathol.1966; 19(5):479-81.

32. Brody GL, Belding AW, Elding MR, Feidman SA: The identification and delineation of myocardial infarcts. Arch Pathol. 1987; 84:312-17.

33. McVie JG: Postmortem detection of inapparent myocardial infarction. J Clin Pathol;23:203-209

34. Mark V. Sherrid Pathophysiology and Treatment of Hypertrophic Cardiomyopathy, Progress in Cardiovascular Diseases, 2006:1-29. 